\title{
Global uniqueness in an inverse problem for a class of damped stochastic plate equations
}

\author{
Qingmei Zhao ${ }^{1}$ and Yongyi $\mathrm{Yu}^{2}$ \\ ${ }^{1}$ Sichuan University \\ ${ }^{2}$ Northeast Normal University
}

May 18, 2021

\begin{abstract}
This paper deals with the global uniqueness of an inverse problem for the stochastic plate with structural damping. The key point is the Carleman estimate for the fourth order stochastic plate operators dyt - p[?]ytdt $+[?] 2 y d t$. To this aim, a weighted point- wise identity for a fourth order stochastic plate operator is established, via which we obtained the desired Carleman estimate for the corresponding stochastic plate equation with structural damping.
\end{abstract}

\section{Hosted file}

4.7.pdf available at https://authorea.com/users/414426/articles/522460-global-uniqueness-inan-inverse-problem-for-a-class-of-damped-stochastic-plate-equations 